\title{
Quand les linguistes et les philologues s'accordent : l'exemple de quelques manuscrits tardifs du Livre de la cité des dames de Christine de Pizan
}

\author{
Andrea VALENTINI \\ Université Sorbonne Nouvelle Paris 3 \\ andrea.valentini@sorbonne-nouvelle.fr \\ https://orcid.org/0000-0002-4654-675X
}

\section{Resumen}

El objeto de este artículo es demostrar, mediante el análisis de un corpus circunscrito (tres manuscritos que contienen una versión anónima del Libro de la ciudad de las damas de Christine de Pizan, versión conocida como V1), que las variantes lingüísticas, tradicionalmente consideradas inútiles para fines filológicos, pueden de hecho proporcionar datos interesantes para el estudio de la tradición manuscrita. Además, cruza el estudio de la estratificación de los diferentes sistemas gráficos de estos manuscritos (su «diasistema») con los datos proporcionados por la filología para explotarlos con el fin de datar y localizar esta versión $V 1$ '.

Palabras clave: sistemas gráficos, diasistema, filología, variantes lingüísticas, Libro de la ciudad de las damas de Christine de Pizan.

\section{Résumé}

La présente contribution voudrait démontrer, par le biais de l'analyse d'un corpus circonscrit (trois manuscrits qui contiennent une version anonyme du Livre de la cité des dames de Christine de Pizan, version connue comme V1), que les variantes linguistiques, traditionnellement considérées comme inutiles à des fins philologiques, peuvent en réalité apporter des données intéressantes à l'étude de la tradition manuscrite. Par ailleurs, elle croise l'étude de la stratification de systèmes graphiques différents dans ces manuscrits (leur "diasystème ») avec les données fournies par la philologie pour les exploiter à des fins de datation et de localisation de cette version $V 1$ '.

Mots-clés : systèmes graphiques, diasystème, philologie, variantes linguistiques, Livre de la cité des dames de Christine de Pizan.

\footnotetext{
*Artículo recibido el 15/09/2020, aceptado el 30/03/2021.
} 


\begin{abstract}
This contribution would like to demonstrate, through the analysis of a delimited corpus (three manuscripts containing an anonymous version of Christine de Pizan's Book of the City of Ladies, version known as VI), that linguistic variants, traditionally considered useless for philological purposes, can in fact provide interesting data for the study of the manuscript tradition. Moreover, it combines the study of the stratification of different graphic systems in these manuscripts (their 'diasystem') with the data provided by philology and codicology in order to exploit them for dating and locating this V1'version.

Keywords: graphic systems, diasystem, philology, linguistic variants, Christine de Pizan's Book of the City of Ladies.
\end{abstract}

\title{
1. Introduction
}

Il est bien connu que les manuscrits copiés à des époques et dans des centres de production différents présentent des variations graphiques diachroniques ou diatopiques plus ou moins marquées. Ce type de variation correspond en grande partie à ce qui a été décrit, surtout pour l'époque de l'ancien français, comme relevant de la scripta (Gossen, 1967). D’autres variations sont liées à des habitudes personnelles des copistes : il a été prouvé que, pour certaines graphies, les copistes pouvaient avoir une préférence pour une forme plutôt que pour une autre, avec des proportions qui peuvent atteindre 100 \% (Cazal, Parussa, Pignatelli et Trachsler, 2003). Plus récemment, il a été avancé de manière convaincante qu'un certain degré de variation peut être le fait d'un choix délibéré des copistes, qui pouvaient appliquer à la graphie le principe de la variatio (Llamas-Pombo, 2017).

Cesare Segre avait indiqué un autre facteur de variation dès les années 1970 : la fidélité des copistes à leur modèle. En empruntant une notion à la sociolinguistique (Weinreich, 1954), il considérait les systèmes linguistiques des manuscrits médiévaux comme un "diasystème", à savoir " un sistema di compromesso tra due sistemi in contatto" (Segre, 1979: 58). Cet auteur soulignait l'importance de prendre en compte l'équilibre toujours instable entre le système du modèle et le système du copiste et faisait remarquer que le modèle pouvait exprimer lui-même un ou plus plusieurs diasystèmes, pouvant être lui-même une copie (d'une copie, d'une copie...). La notion de diasystème a été reprise plus récemment dans les études de stratigraphie linguistique (Greub, 2018).

On peut donc considérer que le diasystème des manuscrits médiévaux est composé d'au moins cinq strates : la fidélité au modèle ; les traits motivés diachroniquement; les usages que, en simplifiant, l'on pourrait définir régionaux; les habitudes personnelles; le goût pour la variatio. Le degré de ce qui est volontaire et de ce qui ne l'est pas dans ces différentes composantes est variable et n'est pas facile à évaluer. Les habitudes personnelles sont probablement les traits les moins conscients chez les 
copistes (Valentini, 2017). Les usages régionaux doivent être aussi employés en grande partie spontanément, bien que les copistes aient pu vouloir rendre leurs textes accessibles au-delà de leur région et avoir ainsi gommé consciemment des traits marqués diatopiquement ${ }^{1}$. La fidélité au modèle peut être aussi plus ou moins intentionnelle. Quant aux innovations diachroniquement motivées, elles peuvent être involontaires, les copistes pouvant moderniser inconsciemment leur modèle ; mais elles peuvent être aussi conscientes, quand elles veulent rendre le texte plus compréhensible pour les lecteurs contemporains, ce qui est davantage vrai pour les imprimés que pour les manuscrits (Parussa, 2019). Par ailleurs, la fidélité au modèle peut être aussi une fidélité marquée diachroniquement dans le sens de l'archaïsme, quand les archaïsmes ne sont pas ajoutés volontairement dans des manuscrits plus récents pour donner une impression d'ancienneté (Pantcheva Capin, 2004). Enfin, le goût pour la variatio semble être l'un des traits les plus réfléchis. Ces strates peuvent être résumées schématiquement de la manière suivante :

Automatismes Habitudes per- Usages régionaux sonnelles

Fidélité au
modèle

Choix intentionnels Innovations Goût pour diachroniquement la variatio motivées

Dans la présente contribution, nous voudrions approfondir l'étude du diasystème dans les manuscrits médiévaux à l'aide d'un corpus homogène. Nous nous intéresserons surtout à la fidélité des copistes à leur modèle et essayerons de montrer que ce qu'on appelle parfois les " variantes linguistiques " peut être d'une grande utilité aux philologues et aux éditeurs des textes médiévaux, alors qu'elles sont encore quelquefois écartées de l'étude de la tradition manuscrite.

Que la distinction traditionnelle entre «variantes de forme » et "variantes de contenu " soit bien plus difficile à établir que ce que l'on a cru autrefois a déjà été soutenu à plusieurs reprises et, de manière limpide, encore assez récemment (Wilhelm, 2013). Dans les pages qui suivent, nous tâcherons de montrer que, dans quelques cas, on peut bien parler de variantes de forme, ou de variantes linguistiques, ou de microvariance, mais que ces variantes peuvent être tout aussi utiles aux linguistes qu'aux philologues : en effet, l'analyse philologique laisse voir que les copistes pouvaient retenir de leur modèle non seulement le contenu (ou les leçons, ou la macro-variance), mais aussi, en partie, la forme, qui est ici à entendre comme système graphique ou morphographique. Cela contribuera à accroître un peu plus notre connaissance de la manière de travailler des copistes, qui reste encore mal connue, ainsi que notre compréhension du

${ }^{1}$ Il est inutile de rappeler que la question de ce qui est régional et de ce qui est commun, ainsi que de ce qui est conscient et de ce qui est involontaire dans les scriptas médiévales est on ne peut plus débattue, et la bibliographie sur ce sujet, pléthorique, ne saurait être résumée ici. Pour une synthèse équilibrée, nous renvoyons à Lusignan (1999). 
degré de conscience linguistique des scripteurs médiévaux. Et nous verrons que, si la linguistique peut fournir des éléments intéressants à la philologie, la philologie, même dans notre ère post-philologique (ainsi dans des traditions universitaires de plus en plus nombreuses), peut aussi être utile à la linguistique.

\section{Le Livre de la cité des dames de Christine de Pizan : manuscrits et éditions}

Les résultats présentés dans cette contribution sont le fruit partiel d'un travail plus ample qui consiste en l'étude de la tradition manuscrite du Livre de la cité des dames de Christine de Pizan (par la suite $C D$ ) et en l'édition critico-génétique d'une partie de ce texte ${ }^{2}$. Nous rappelons que cette œuvre majeure de la littérature médiévale et de la littérature féministe est connue essentiellement en traduction ${ }^{3}$. Le texte original a été édité dans deux thèses de doctorat restées toutes deux inédites (Lange, 1974; Curnow, 1975). Leurs auteures ont étudié, de manière plus ou moins approfondie, les nombreux manuscrits qui conservent ce texte ; mais Langue (1974) n'a utilisé que deux manuscrits pour son édition (le manuscrit de base est $\mathrm{R}$, et le relevé des variantes $\mathrm{du}$ deuxième manuscrit, A, est aléatoire ${ }^{4}$ ) ; Curnow (1975), quant à elle, s'est servie de trois manuscrits ( $\mathrm{D}$, avec les variantes de $\mathrm{R}$ et de $\mathrm{B})$.

Le texte en moyen français de la $C D$ n'a été publié qu'en 1997 (à lire aujourd'hui dans Caraffi et Richards, 2003), d'après le même $\mathrm{R}$ utilisé par Langue (1974) : ce codex, connu comme le "manuscrit de la Reine ", est un très luxueux recueil de ses œuvres que Christine de Pizan a offert à la reine de France Isabelle de Bavière après 1410, probablement en 1414 . Dans l'édition Caraffi et Richards (2003), on trouve un choix très restreint (sept pages pour environ 230 pages de texte) de variantes de trois manuscrits $(\mathrm{A}, \mathrm{D}$ et $\mathrm{P} 3)$. Depuis plusieurs années est en préparation une nouvelle édition de la $C D$ avec une traduction en français moderne en regard, par Anne Paupert et Claire Le Ninan, d'après un manuscrit inédit (P3), avec les variantes de $\mathrm{R}$; l'édition devrait comporter également un choix de variantes de deux autres témoins (B et D) ${ }^{6}$. La $C D$ se lit aujourd'hui aussi en regard de l'édition de la traduction anglaise que Brian Anslay en a publié en 1521 : son éditrice, Johnston (2014), a en effet eu l'heureuse initiative de transcrire aussi le texte dont le traducteur s'est sans doute servi (Lo), avec les variantes du même R plusieurs fois cité. Comme on le voit, il s'agit à chaque fois d'éditions qui prennent en compte un nombre très limité de manuscrits, dont elles n'offrent d'ailleurs le plus souvent qu'un choix de variantes.

${ }^{2}$ "Or est du tout achevee et parfaitte nostre cité ": Élaboration linguistique et genèse du texte dans le Livre de la cité des dames de Christine de Pizan (à paraître).

${ }^{3}$ Pour une étude récente du point de vue des études de genre, publiée dans cette même revue, voir Ibeas Vuelta (2020).

${ }^{4}$ Pour les sigles des manuscrits de la $C D$, on se reportera à l'Annexe 1.

${ }^{5}$ Un site internet est entièrement consacré à ce manuscrit : http://www.pizan.lib.ed.ac.uk.

${ }^{6}$ Nous tenons ces informations des éditrices, que nous remercions. 
La tradition manuscrite de la $C D$ se compose de vingt-sept manuscrits plus un fragment, d'un feuillet seulement, d'un vingt-huitième. À ces témoins il faut ajouter la traduction anglaise qui n'existe que dans l'édition citée de 1521 et une traduction flamande qui subsiste dans un manuscrit. Sept de ces témoins, ainsi que le fragment, sont ce qu'on appelle des " manuscrits originaux » $(\mathrm{MO})$, à entendre comme des copies préparées par un petit groupe de copistes sous le contrôle de l'auteure pour les offrir à des mécènes, dont certains sont connus ; Christine de Pizan intervenait probablement ellemême si ce n'est dans la copie, au moins dans la relecture et la correction de ces manuscrits, qui peuvent être datés d'entre 1405 et 1415 environ. Ces manuscrits ont été étudiés dans Reno (2006 ; repris et légèrement modifié dans Ouy, Reno et VillelaPetit, 2012 : 517-528). Toutes les éditions mentionnées ci-dessus ont utilisé ces manuscrits, à l'exception de Johnston (2014), mais certains MO n'ont jamais été pris en considération ${ }^{7}$.

Les vingt manuscrits restants ont été produits indépendamment du contrôle de l'auteure, déjà les plus anciens sans doute après sa mort ; nous les appellerons " manuscrits tardifs" (MT). Jusqu'à récemment, ces manuscrits n’avaient jamais été étudiés, sauf dans les deux thèses inédites. Les descriptions codicologiques de Curnow (1975: 346-546) sont extrêmement précieuses, mais les résultats philologiques auxquels elle aboutit ne peuvent pas être acceptés (570-592) ; c'est néanmoins cette chercheuse qui a eu le mérite de reconnaître dans un MT, Lo, la source de la traduction anglaise (334). Quant à Lange (1974), elle s'intéresse plus particulièrement aux MO (XL-LXV), mais elle fait aussi une place, bien que très limitée, aux MT (LXVI-LXVIII) ${ }^{8}$. Ceux-ci nous fournissent des indications particulièrement intéressantes sur l'évolution et sur la diffusion du texte, mais également sur sa genèse (Valentini, 2019). Les MT offrent aussi une mine d'informations d'un point de vue linguistique, alors que jusqu'à présent la langue avait été presque totalement occultée dans les études sur ce texte à la tradition foisonnante?

\footnotetext{
${ }^{7}$ Comme nous l'avons vu, les éditions utilisent, à différents degrés, $\mathrm{A}, \mathrm{B}, \mathrm{D}, \mathrm{P} 3$ et R ; les MO qui n'ont jamais été pris en compte sont P1 et P2, comme le fragment L.

${ }^{8}$ Par ailleurs, cette thèse n'est pas consultable, pas même en s'adressant à l'Université de Hambourg (courriels des 4 et 7 novembre 2016 de la part des responsables respectivement de la Bibliothèque de l'Université de Hambourg et de la Bibliothèque du département Sprache, Literatur, Medien). Nous avons pu la consulter dans sa totalité grâce à l'obligeance d'Anne Paupert, qui a mis à notre disposition sa copie personnelle. Les résultats les plus importants atteints par Lange (1974) ont été résumés, mais avec quelques imprécisions, dans Richards (2000 : 791 et passim).

9 À l'exception notable de Brown-Grant (2000) et Brown-Grant (2002), qui s'est intéressée aux procédés linguistiques adoptés par Christine de Pizan dans sa récriture des passages qu'elle a empruntés à l'une de ses sources principales, le Des cleres et nobles femmes, la traduction anonyme du De mulieribus claris de Boccace.
} 
Nous avons collationné les manuscrits de la $C D$ par loci selecti ${ }^{10}$. La collation a mis en lumière l'existence de quatre versions : les deux premières (que nous avons baptisées $V 1$ et $V 2$ ) remontent assurément à l'auteure, qui a remanié la $V 1$ après en avoir fait tirer certaines copies. Elles sont conservées à la fois dans des $\mathrm{MO}$ et dans des $\mathrm{MT}^{11}$. Une version proche de la $V 1$ est conservée uniquement dans trois MT (V1) : un examen stylistique et linguistique a démontré qu'il s'agit d'un remaniement que l'on ne peut pas attribuer à Christine de $\operatorname{Pizan}^{12}$. La quatrième version n'en est pas vraiment une, en ce sens qu'elle est le fruit d'une contamination de cette $V 1$ ' et de la $V 2$, mais le copiste à l'origine de son archétype a, de surcroît, une forte tendance à innover (cette rédaction a été appelée $V x$ ). Au demeurant, c'est la version qui est conservée dans le plus grand nombre de $\mathrm{MT}^{13}$, ce qui confirme, si besoin est, que les notions d'" original » et de «dernière copie surveillée par l'auteur.e » peuvent être peu pertinentes pour le Moyen Âge.

\subsection{Corpus et objectifs}

Dans la présente contribution, nous nous intéresserons à la rédaction que nous avons appelée $V 1$ '. Comme il a été anticipé, cette version est conservée dans trois MT: $\mathrm{Mu}, \mathrm{Pa} 1$ et Wi. La production des deux derniers peut raisonnablement être datée des années 1460-1470 et être située en Normandie, probablement à Rouen, comme le suggère leur iconographie. $\mathrm{Mu}$, quant à lui, a sans doute été produit dans le deuxième quart du XV $\mathrm{XV}^{\mathrm{e}}$ siècle, et plus probablement dans les années 1440, à Paris ou possiblement aussi en Normandie ${ }^{14}$.

\footnotetext{
${ }^{10} \mathrm{La} C D$ comporte trois parties qui se composent respectivement, dans les MO les plus récents, de 48, de 69 et de 19 chapitres. Les chapitres qui ont été collationnés en entier sur un certain nombre de manuscrits - tous les MO, plus Lo, Mu et Pa2 parmi les MT - sont les suivants : 1.1-1.4, 1.21-1.25, 2.12, 2.29, 2.53-2.54, 2.61-2.64, 3.14-3.15, 3.19, plus les tables des matières. Les autres MT ont été collationnés sur des portions de texte plus restreintes (voir ci-dessous pour le corpus retenu dans l'étude de $\mathrm{Pa}$ l et de Wi). Des sondages ont été réalisés aussi dans d'autres chapitres du texte dans tous les manuscrits.

${ }^{11}$ Respectivement, la $V 1$ dans deux $\mathrm{MO}$ et dans deux MT, ainsi que dans la traduction anglaise, tirée de l'un de ces derniers; la V2 dans cinq MO (ainsi que dans le fragment) et dans six MT. Pour la liste des versions conservées dans les différents manuscrits, on se reportera à l'Annexe 1.

${ }^{12}$ Une étude sur les procédés à l'œuvre lors du remaniement $V 1$ 'n'a pas encore été publiée. Pour l'instant, on pourra au moins consulter les notes apposées à l'extrait d'édition synoptique (V1 / V1'/ V2) publié dans Valentini (2019: 437-445).

${ }^{13}$ Huit, ainsi que dans la traduction flamande. Pour tout ceci, ainsi que pour le choix des loci selecti, on se reportera pour le moment à Valentini (2019). L'ouvrage signalé dans la note 2 apportera quelques rectifications à certains points traités dans cet article, et les loci selecti y ont été augmentés par rapport à celui-ci.

${ }^{14}$ Il n'a pas été possible d'insérer dans la présente contribution une étude codicologique : il existe actuellement une étude fiable publiée uniquement de Wi (Pächt et Thoss, 1974 : 1, 52-53). Quant à Pal et à $\mathrm{Mu}$, pour l'instant on pourra se reporter à ce qui a été écrit dans Valentini (2019: 427-428). Un
} 
Comme nous l'avons écrit rapidement ci-dessus, la $V 1$ ' a été copiée aussi dans un manuscrit dans lequel elle a été croisée avec la $V 2$ (la version de la $C D$ revue par Christine de Pizan) : c'est le manuscrit à l'origine de la $V x$. La contamination rend difficile la collation entre les témoins de la $V 1$ 'et les témoins de la $V x$, et les manuscrits de cette version ne seront donc pas étudiés systématiquement dans la présente contribution; ils seront néanmoins mis à profit le moment venu car, comme nous le verrons, ils peuvent fournir des éléments de comparaison intéressants. Avant de passer au cœur de l'analyse linguistique, nous dirons toutefois que le manuscrit utilisé pour produire cette $V x$ était vraisemblablement $\mathrm{Mu}$ lui-même ou un manuscrit copié d'après ce dernier.

$\mathrm{Mu}$, le témoin le plus ancien de la $V 1$ ', ne peut en aucun cas être considéré comme l'archétype de celle-ci, étant donné que non seulement il fait état de quelques innovations qui lui sont tout à fait singulières, et qui sont passées dans la $V x$, mais aussi il partage assurément un intermédiaire commun avec Pa1. On peut être certain de ces affirmations, car il y a des cas dans lesquels Pal et Wi suivent la $V 1$, la version " originale ", contre Mu seul (et les témoins de la $V x$ ); mais il y a aussi des cas où Wi seul suit la $V 1$ contre Mu et Pal (et les témoins de la $V x$ ). Toutefois, Wi, bien qu'isolé, reste un manuscrit très peu fiable, car son copiste a une forte tendance à innover, surtout en banalisant et en écourtant le texte.

Il n'est pas possible de fournir ici tous les éléments qui nous ont permis d'arriver à ces conclusions, mais nous pouvons citer au moins deux exemples particulièrement éclairants. Dans le chapitre 2.29, où il est question d'Antonina et de son mari Bélisaire, dans $\mathrm{Mu}$ et dans $\mathrm{Pa} 1$ on lit :

Et je auray l'autre partie de voz gens avecques moy et arriverons de l'autre part au port. Et tandis qu'ilz entendront à vous donner la bataille, nous entrerons de l'autre $\dagger$ es villes et chasteaux et metrons tout à mort, à feu et à flambe (graphie de Mu f. 61rb ; Pal f. 57r).

Les témoins de la $V x$ offrent la même leçon : pour cette contribution, nous nous contenterons de citer un seul témoin, $\mathrm{Pa} 2$ (France du Nord, troisième quart du $\mathrm{XV}^{\mathrm{e}}$ siècle, f. $78 \mathrm{rb}$ ). L'énoncé n'a pas beaucoup de sens, car il manque un mot où nous avons placé une crux, part, qu'on lit dans le texte non remanié, tout comme dans Wi, qui présente toutefois d'autres innovations à cet endroit (le texte original a par ailleurs été en partie modifié dans la $V 1)$ :

Et je raray [je r. manque Wi] l'autre partie de voz gens avec moy et par mer [p. m. manque Wi] arriverons de l'autre part au port, et tandis que ilz entendront à vous donner la bataille, nous entrerons de l'autre part es villes et

quatrième manuscrit, dont on a perdu la trace, pourrait appartenir à cette version ; il n'est pas impossible qu'il ait été produit en Normandie (Valentini, 2019 : 202, note 31, et 429, note 89). 
es citez [et chasteaulx $\mathrm{Wi}$ ] et mettrons tout à mort et [et manque $\mathrm{Wi}$ ] à feu et flamme (d'après A [VI] f. 77vb-78ra ${ }^{15}$; Wi f. 94v).

L'absence de ce mot est clairement un bourdon, à interpréter comme une erreur conjonctive entre $\mathrm{Mu}$ et Pa1. Elle est appuyée par une série de variantes communes à ces deux manuscrits.

$\mathrm{Pa}$ l partage donc un antigraphe avec $\mathrm{Mu}$, mais il n'en est pas un descriptus. Dans le chapitre 1.1, on trouve un énoncé complexe, où Pa1 suit l'original :

Mais non obstant que, pour chose que je peusse congnoistre, tant longuement y sceusse visiter [viser P1 Lo Pa1] ne esplucher, je ne apperceusse ne [et Pa1] congeusse telz jugemens estre vrays... (A [Vl] f. 1vb ; Pa1 f. 2v).

Mu offre ici une leçon corrompue par une erreur d'anticipation (en italique ciaprès) ; $\mathrm{Pa} 2$, le témoin de la $V x$, comporte une faute supplémentaire, mais qui dérive clairement de la leçon de $\mathrm{Mu}$ (je y sceusse > il y sceussent] :

Mais non obstant que [manque Pa2], pour chose que je y sceusse [il y sceussent $\mathrm{Pa} 2$ ] congnoistre, tant longuement y sceusse viser ne espelucier, je ne apperceusse ne congneusse telz jugemens estre vrais... (Mu f. 4vb ; Pa2 f. 5ra).

$\mathrm{Wi}$, quant à lui, innove de manière indépendante :

Et combien que je ne sceusse si bien espelucher ne viser que ce trouvasse estre vray (f. 4r).

Un stemma codicum simplifié de la V1'est fourni dans l'Annexe 2. Cette rapide présentation des données ne serait pas complète si nous ne disions pas que le copiste à l'origine de la $V 1$ ' a travaillé sur un manuscrit certes de la première version de la $C D$ (V1), mais qui devait être légèrement postérieur à tous les manuscrits conservés de cette version, car la rédaction remaniée fait état de quelques innovations qui n'apparaissent que dans la V2: l'auteure avait donc dû apporter sur ce manuscrit ou sur son modèle quelques modifications en vue de la deuxième version de son texte. Puisque le manuscrit le plus ancien de la V2 (P2) ne semble pas pouvoir être daté d'avant 1406 et que la $V 1$ n'a pas été complétée avant 1405, le manuscrit utilisé par le remanieur doit avoir été produit dans cet intervalle ${ }^{16}$.

Le scribe à l'origine de la $V 1$ ' a donc dû copier l'archétype de cette rédaction entre 1405, terminus post quem pour la production des premiers MO de la $C D$, et 1450 environ, terminus ad quem pour la production de Mu. Cette fourchette d'un demi-

\footnotetext{
${ }^{15}$ Dans la V2, ce passage est identique : voir Caraffi et Richards (2003 : 292).

${ }^{16}$ La rédaction de la première version de la $C D$ est traditionnellement située en 1404-1405 (voir Solente, 1969 : 47, qui se fonde sur des arguments, contestables, de Laigle, 1912 : 16-17). Nous croyons qu'elle a plutôt été composée en 1405-1406, mais il serait trop long d'en faire ici la démonstration. De la même manière, il n'est pas possible de donner plus de précisions sur la datation des $\mathrm{MO}$ dans la présente contribution. Dans Ouy, Reno et Villela-Petit (2012 : 545), P2 est daté de 1405-1406, mais la date de 1405 nous semble à exclure pour le remaniement $V 2$.
} 
siècle peut-elle être restreinte grâce aux données fournies par l'analyse linguistique ? Une approche diachronique peut-elle nous aider dans cette opération ? Par ailleurs, estil possible de localiser le remaniement grâce à l'étude de la langue ? Plus particulièrement, la linguistique variationnelle peut-elle nous dire si le copiste-remanieur à l'origine de la $V 1$ 'était lui-même normand, comme normands sont certainement deux des trois témoins de cette version, et peut-être aussi le troisième ?

\section{Les « variantes linguistiques" des manuscrits de la version VI'}

Pour la présente contribution, nous avons utilisé un corpus restreint, facilement maîtrisable pour une comparaison entre quatre manuscrits ${ }^{17}$ et qui offre déjà des résultats satisfaisants. Il est constitué des chapitres suivants de la $C D$ : 1.1-1.2, 1.21-1.22, 2.29, 2.53, 2.63, 3.19. Des sondages ont été réalisés dans d'autres parties du texte. Nous devons préciser que ces chapitres ne correspondent pas toujours aux chapitres qui portent les mêmes numéros d'ordre dans l'édition publiée dans Caraffi et Richards (2003), car celle-ci se fonde sur la version la plus récente du texte (V2), alors que le remaniement qui est ici étudié est basé sur la première version du texte $(V I)^{18}$. Pour ces raisons, la $V 1$ ' sera toujours comparée à l'édition de la $V 1$ que nous avons nousmême procurée, d'après le manuscrit $\mathrm{A}^{19}$.

\subsection{Le remplacement de mie par pas : une idiosyncrasie de copiste ?}

En comparant les chapitres de la $V 1$ 'édités d'après $\mathrm{Mu}$ avec les mêmes chapitres de la $V 1$ d'après $\mathrm{A}$, on observe qu'il arrive que la $V 1$ ' utilise l'adverbe négatif pas où la $V 1$ emploie mie. Mais ce remplacement est localisé uniquement dans un endroit de notre corpus, dans le chap. 2.53 : mie peut former un syntagme avec non (non mie > non pas) ou il peut être le deuxième morphème de la négation "complète " (ne... mie $>n e . . p a s)$. Le remplacement n'est d'ailleurs pas systématique; on l'observe dans cinq des sept occurrences de mie de la $V 1^{20}$ :

Tu pués veoir par ce que devant t'ay dit que des dames, dont je t'ay raconté les grans vertus cy dessus, en diverses oeuvres differenciees l'une de l'autre leur

\footnotetext{
${ }^{17} \mathrm{Mu}$, Pa1 et Wi pour la $V 1$ 'et $\mathrm{Pa} 2$ pour la $V x$, auxquels s'ajouteront, comme on le verra au cas par cas, des manuscrits variables de la $V 1$, de la $V 2$ et de la $V x$.

${ }^{18}$ Le décalage le plus important se situe à la fin de la deuxième partie, car le chap. 2.53 de la $V 1$ (et de la $V 1$ ) a été scindé en deux dans la $V 2$ (> 2.53-54); ainsi, le chap. 2.63 de notre corpus correspond au chap. 2.64 de la $V 2$.

${ }^{19} \mathrm{Il}$ y a néanmoins un décalage aussi entre ce manuscrit et les témoins de la $V 1$ ', car dans les manuscrits de la $V 1$ arrivés jusqu'à nous le chapitre consacré à Artémise, reine d'Halicarnasse, est le $\mathrm{n}^{\circ} 25$ de la première partie, mais dans la $V 1^{\prime}$ il occupe la place 21, comme plus tard dans la $V 2$ : c'est l'un des éléments qui font penser que le manuscrit utilisé par le remanieur était postérieur aux manuscrits conservés de la V1. Nous rappelons que la $V 1$ peut être consultée aussi dans Johnston (2014) mais, en présence de $\mathrm{MO}$, nous avons préféré ne pas renvoyer à cette édition établie d'après un MT.

${ }^{20}$ Seule sera mise en évidence la différence en question (mie > pas). Les autres modifications propres à la $V 1$ ' ne seront pas commentées ici.
} 
entendement occupoient, non mie toutes en une meismes chose. Ceste oeuvre estoit à bastir à toy reservee et non mie à elles... $\left(2.53 .7-8^{21}: \mathrm{A}[\mathrm{VI}], \mathrm{f} .102 \mathrm{vb}-\right.$ 103ra).

Tu peuz veoir par ce que devant t'ay dit que des dames, dont je t'ay raconté cy dessus les grans vertus, en divers oeuvres differenciéz l'un de l'autre leur entendement occupoient, et non pas toutes en une mesmes chose. Ceste oeuvre à bastir estoit à toy reservee et non pas à elles... (2.53.7-8: Mu [V1'], f. $77 \mathrm{va})$.

...pour ce que la matiere, quoy que elle soit fondee sur loy de nature, ne vient mie du tout par l'attrempeement de raison (2.53.21 : A [V1], f. 103rb).

...pour ce que la matiere, quoy qu'elle soit fondee sur loy de nature, ne vient pas du tout par l'attrempeement de raison (2.53.21: Mu [V1'], f. 78ra).

Mais sur le point que tu m'as touchié que ilz dient que pour le bien commun le font, je te monstreray que pour ce ne fu ce mie [...]. Et n'est mie doubte que les femmes sont aussi bien ou nombre de creature humaine que sont les hommes (2.53.31 et 2.53.35: A [VI], f. 103vb-104ra).

Mais sur le point que tu m'as touché que pour le bien commun le firent, je monstreray que pour ce ne fu ce pas [...]. Et n'est pas doubte que les femmes sont aussi bien ou nombre de creature humaine comme sont les hommes (2.53.31 et 2.53.35: $\mathrm{Mu}[V 1$ '], f. 78rb-78va).

Dans les autres chapitres et à deux reprises dans ce même chap. 2.53, le morphème mie est gardé, aussi bien en composition avec non (sept fois ${ }^{22}$ ) que pour compléter l'adverbe négatif $n e$ (cinq fois ${ }^{23}$ ). Dans quelques cas, pas est déjà dans la V1 (sept fois $\left.{ }^{24}\right)$.

Mais le remplacement de mie par pas ne peut pas être attribué à la personne à l'origine de la $V 1$ '. Les deux autres témoins de cette rédaction, Pa1 et $\mathrm{Wi}$, gardent l'adverbe mie de la $V 1$ où $\mathrm{Mu}$ le remplace par pas (le chap. 2.53 se lit aux f. $72 \mathrm{v}-74 \mathrm{r}$ dans Pal et aux f. 123v-125v dans Wi). La modification est donc à attribuer au copiste de $\mathrm{Mu}$, ou au copiste d'un antigraphe perdu intermédiaire entre ce manuscrit et le modèle qu'il partage avec Pa1.

Un manuscrit perdu est peut-être à postuler car, dans $\mathrm{Mu}$, on n'observe pas de différence d'écriture à l'endroit du texte où se trouve le chap. 2.53, alors qu'un changement de copiste dans un hypothétique antigraphe pourrait expliquer que mie soit remplacé par pas uniquement dans ce chapitre de notre corpus. Les graphies aussi semblent assez stables d'un bout à l'autre de $\mathrm{Mu}$, mais le corpus n'offre pas suffisamment

${ }^{21}$ Ces subdivisions renvoient aux paragraphes dans lesquels a été divisé le texte dans l'édition signalée dans la note 2 ci-dessus.

${ }^{22} \S 1.1 .3,1.1 .9,1.2 .19,1.2 .25,2.53 .24,2.53 .33,3.19 .5$.

${ }^{23} \$ 1.1 .20,1.2 .7,1.25 .4 V 1=1.21 .4 V 1,1.25 .19 V 1=1.21 .19 V 1,3.19 .7$.

${ }^{24} \S 1.1 .7,1.2 .15,2.29 .24,2.53 .13,2.63 .2,2.63 .4,3.19 .8$. 
d'exemples pour l'affirmer avec certitude. La vérification a été faite sur les graphies des mots peut, hault, sans et des suffixes -esche, -eulzl-eux (les graphies retenues sont celles que le copiste de $\mathrm{Mu}$ préfère, plutôt que, par ex., puet, haut, sanz, -ece, -eulx). Seules les graphies sans et -eulz /-eux donnent des indications satisfaisantes, car elles reviennent à la fois dans le chap. 2.53 et ailleurs : dans $\mathrm{Mu}$ on trouve sans de manière constante (huit fois ${ }^{25}$ ) ; pour noter le masculin pluriel du pronom personnel complément et du pronom démonstratif en -l- (eulz, (i)ceulz), la graphie employée est toujours-eulz (dix-huit fois ${ }^{26}$ ); pour noter le suffixe des adjectifs formés avec le suffixe qui continue le latin -ŌSUS, ainsi que les continuateurs de DEUS, DUO, LOCUS, MELIUS et PRODE, la graphie employée est toujours -eux (vingt-neuf fois ${ }^{27}$ ).

L'adverbe pas est de plus en plus courant au $\mathrm{XV}^{\mathrm{e}}$ siècle : les rapports avec mie, qui était plus courant jusqu'au début du XIV $\mathrm{XI}^{\mathrm{e}}$ siècle, s'inversent et, après une période de concurrence, pas finira par remplacer mie (Marchello-Nizia, 1997 : 304). La modification ici étudiée, sans doute involontaire, n'a donc rien d'étonnant sous la plume d'un copiste du deuxième quart du XV $\mathrm{XV}^{\mathrm{e}}$ siècle, que ce copiste soit à identifier avec celui de $\mathrm{Mu}$ ou avec celui d'un antigraphe qui aurait pu être de peu antérieur.

Quoi qu'il en soit, ce remplacement semble bien relever d'un manuscrit en particulier plutôt que de la rédaction $V 1$ '. Il peut porter une pièce d'appui supplémentaire à un élément déjà connu, le remplacement progressif de mie par pas en français au cours du moyen français, mais ne peut pas nous aider à dater le remaniement VI'. Par ailleurs, l'imposition progressive de pas sans doute dès la première moitié du $\mathrm{XV}^{\mathrm{e}}$ siècle à l'écrit, voire vraisemblablement plus tôt à l'oral, n'a pas empêché les copistes de Pa1 et de Wi, dans le troisième quart du siècle, de rester fidèles à leurs modèles et d'écrire mie. Nous faisons enfin remarquer que les manuscrits de la $V x$ ont pas là où l'on trouve cet adverbe dans $\mathrm{Mu}^{28}$, à confirmation du fait que le manuscrit utilisé par le copiste à l'origine de la contamination entre la $V 2$ et la $V 1$ ' avait sous les yeux $\mathrm{Mu}$ ou un manuscrit très proche de celui-ci.

\subsection{Le remplacement de comment par comme : une idiosyncrasie de remanieur ?}

Si le remplacement de mie par pas ne semble pas pouvoir être attribué à la personne à l'origine de la $V 1$ ', d'autres changements peuvent à coup sûr lui être imputés. Ainsi du remplacement de l'adverbe interrogatif comment par comme. Le procédé est

${ }^{25} \$ 1.1 .10,1.21 .8,1.21 .11,2.53 .4,2.53 .8,2.53 .9,2.53 .11,3.19 .12$.

${ }^{26}$ (I)ceulz $\$ 1.1 .16,1.2 .8,1.2 .20,1.21 .6,1.21 .8$ (deux fois), 1.21.10 (deux fois), 1.21.12, 1.21.14, $1.21 .16,2.29 .2$, 3.19.10, 3.19.17 ; eulz $\$ 1.21 .15,2.53 .18,2.53 .24,2.53 .27$.

27 Angoisseux $\$ 3.19 .22$; deux $\$ 1.1 .9,1.21 .5,1.21 .8,1.21 .18,2.29 .21,2.53 .2,2.53 .36$; Dieux $\$ 3.19 .2$; envieux $\$ 2.29 .16$; honteux $\$ 1.22 .13$; joyeux $\$ 2.29 .18$; lieux $₫ 1.1 .11$, 1.2 .17 ; mieux $\$ 1.1 .25,1.2 .8,1.22 .9,2.29 .23,2.53 .2,2.53 .27,2.63 .2$ (deux fois), 2.63.3; orgueilleux $\$ 3.19 .7$; preux $\$ 1.21 .2,1.21 .9,1.22 .2 ;$ vertueux $\$ 1.22 .4 ;$ victorieux $\$ 1.21 .13$.

${ }^{28} \mathrm{La}$ vérification a été faite dans le chap. 2.53 des manuscrits suivants, qui appartiennent à trois groupes distincts de cette rédaction mixte : Pa2 f. 102ra-103vb, Pr f. 151r-153r et Pa8 f. 79r-80v. 
systématique, et les témoins de la $V 1$ 's' accordent entre eux. Dans notre corpus, on ne l'observe pas moins de dix fois ${ }^{29}$, comme par exemple dans les deux passages suivants, où l'adverbe introduit respectivement une interrogative indirecte et une interrogative directe :

...je determinoie que vile chose fist Dieux quant il fourma femme, en m'esmerveillant comment sy digne ouvrier daigna oncques faire tant abhominable ouvrage... (1.1.16: A [V1], f. 2rb).

...je determinoie que vile chose fist Dieu quant il forma femme, en moy merveillant comme si digne ouvrier voulu oncques faire tant abhominable ouvrage... (1.1.16: Mu [V1'], f. 5ra).

Ha ! Dieux, comment puet ce cy estre? (1.1.19: A [VI], f. 2rb).

Ha ! Dieu, come peut cecy estre? (1.1.19: Mu [V1'], f. 5rb).

On rencontre un seul cas où comment est maintenu dans $\mathrm{Mu}$ :

Comment, belle fille, et qu'est ton sens devenu ? (1.2.9 : A [V1], f. 3rb $=\mathrm{Mu}$ [V1], f. 6ra).

Mais si Mu et Wi (f. 5v) présentent ce morphème, Pa1 a la forme comme (f. 3v). $\mathrm{Mu}$ et $\mathrm{Wi}$, dont l'indépendance respective a été établie, auraient-ils réintroduit l'adverbe originel spontanément, peut-être parce qu'il semblait plus naturel à leurs copistes ? Ou bien le copiste de $\mathrm{Pa} 1$, qui, rappelons-le, a un ancêtre commun avec $\mathrm{Mu}$, aurait-il apporté cette modification en allant dans le même sens que le remanieur ?

Ce remplacement systématique méritait d'être signalé, mais les deux morphèmes comme et comment semblent avoir été employés indifféremment jusqu'au XVII ${ }^{e}$ siècle (Marchello-Nizia, 1997 : 215-216). C'est peut-être seulement la condamnation des grammairiens qui a fini par faire reculer l'usage de comme avec la fonction d'adverbe interrogatif (Fournier, $1998: 125, \$ 178$ ). Cette modification ne peut donc pas être utilisée pour dater le remaniement.

\subsection{Le remplacement de tout soit par tant soit : un ténu indice diachronique ?}

Un autre remplacement morpholexical, en revanche, peut être interprété non pas comme une simple idiosyncrasie, mais comme un trait de langue plus "moderne ». Dans tous les témoins de la $V 1$, on lit tant à la place de tout dans les tournures concessives paratactiques du type tant soit... Dans notre corpus, on en trouve deux seuls exemples, mais des échantillons de texte plus vastes qui ont été vérifiés montrent que ce remplacement revient aussi ailleurs dans la $V 1^{\prime}$ :

Mais la veue d'icellui dit livre, tout soit il de nulle auttorité, ot engendré en moy nouvelle pensee... (1.1.9 : A [VI], f. 1va).

Mais la veue d'icelui livre, tant soit il de nulle auctorité, olt engendree nouvele pensee en moy... (1.1.9: Mu [V1] , f. 4va).

${ }^{29} \$ 1.1 .16,1.1 .19,1.1 .22,1.2 .1$ (deux fois), 2.29.7, 2.53.4, 2.53.10, 2.63.1, 3.19.15. 
Et notes de rechief se saint Augustin et autres dotteurs de l'eglise ont point repris meismement Aristote en aucunes pars, tout soit il dit le prince des philosophes... (1.2.13: A [VI], f. 3rb-3va).

Et note derechief se saint Augustin et autres docteurs de l'eglise ont point reprins mesmement Aristote en aucunes pars, tant soit il dit le prince des philozophes... (1.2.13: Mu [V1], f. 6ra).

Les deux tours tout soit et tant soit étaient équivalents en moyen français, selon Marchello-Nizia (1997 : 428-429), qui en parle toutefois rapidement ; l'auteure ellemême cite un exemple avec tant (tiré du Pathelin). Selon Soutet (1992 : 44), " les concessives commençant par tout [...] ne paraissent pas dépasser le début du XV siècle ", et d'ajouter que Christine de Pizan est la dernière auteure de son corpus à en offrir des exemples. La tournure avec tant est restée vivante plus longtemps : on en trouve encore des exemples au début du XVII ${ }^{\mathrm{e}}$ siècle (Fournier, $1998: 363$, $\$ 544$, qui la cite toutefois comme un archaïsme de Malherbe, et avec accord du verbe au pluriel tant soient$i l s)$.

Certes, la plus grande "modernité " de ce trait ne suffit pas à prouver que le remanieur a travaillé des décennies après Christine de Pizan, étant donné que celle-ci pouvait parfois rechercher une langue sans doute déjà archaïsante à son époque (Dugaz, 2019 : 33 et passim). Mais il est vrai qu'un remplacement systématique s'expliquerait mieux s'il avait été opéré plus avant dans le $\mathrm{XV}^{\mathrm{e}}$ siècle, quand la tournure tout soit était devenue trop vieillie.

\subsection{Le suffixe -ore: diatopie d'un antigraphe}

Si de l'étude de mots grammaticaux comme ceux que nous avons vus jusqu'à présent, nous passons à l'étude des graphies, nous pouvons observer que, dans le corpus étudié, toutes les fois que la $V 1$ a un mot terminé en -oire, ce mot est terminé par le suffixe -ore dans l'édition interprétative de la $V 1$ 'préparée d'après $\mathrm{Mu}$.

C'est le cas pour le mot victoires (vittoires dans le manuscrit de base de la V1, A, qui privilégie la ligature $t t$ à $c t$ ), comme dans le passage suivant :

Sy ot avec ce sy grant hardiesce en fait de chevalerie et tant bien en garda la discipline que, par pluseurs vittoires que elle ot, la magesté de son nom par grant renommee tres hault esleva... (1.25.5 : A [VI], f. 32va).

Si olt avec ce si grant hardiesce en fait de chevalerie et si bien en garda la discipline que, par plusieurs victores qu'elle olt, la majesté de son nom par grant renommee tres hault esleva... (1.21.5 : Mu [VI'], f. 25va).

Ce mot, au singulier ou au pluriel, est employé sept fois dans notre corpus ${ }^{30}$, et $\mathrm{Mu}$ écrit constamment victore(s) là où, dans tous les manuscrits de la $V 1$ arrivés jusqu’à

\footnotetext{
${ }^{30}$ En plus du passage cité, dans les $₫ 1.21 .12,1.21 .13,1.21 .26$ (correspondant à $V 1$ 1.25.12, 1.25 .13 et 1.25.26), 1.22.15 (V1 1.21.15), 2.29.23, 3.19.4
} 
nous, et pas uniquement dans A donc, la graphie est toujours vic-/vittoire $(s)^{31}$. On observe le même phénomène avec le mot gloire, qui revient deux fois dans notre corpus, ainsi qu'avec le nom propre Gregoire, employé une fois :

Sy desservéz los et gloire et mes grans tresors ne vous seront espargniéz (1.25.23: A [V1], f. 33va).

Si desservéz los et glore et mes grans tresors ne vous seront espargniéz (1.21.23: Mu [V1], f. 26rb).

Or est eschevee du tout et parfaicte nostre cité, en la quelle à grant honneur vous toutes, celles qui améz gloire, vertu et loz, pouéz estre hebergees... (3.19.3 : A [V1], f. 139va).

Or est nostre cité du tout achevee et parfaicte, en laquele à grant honneur vous toutes, celles qui améz glore, vertu et loz, pouéz estre logees et herbegees ${ }^{32} \ldots$ (3.19.3: Mu [VI'], f. 106ra).

...car dit saint Gregoire que pacience est entree de Paradis et la voie de Jhesucrist (3.19.11: A [V1], f. 140ra-140rb).

...car dit saint Gregore que pacience est entree de Paradis et la voye de Jhesucrist (3.19.11: Mu [V1'], f. 106va).

Ce sont les seuls mots se terminant par le suffixe -oire(s) (dans la graphie « commune ") du corpus, mais, pour tous ces mots, la graphie -ore(s) est constante dans $\mathrm{Mu}$. Vérification faite, Pa1 emploie aussi toujours la graphie -ore $(s)^{33}$, mais, dans Wi, le suffixe est constamment écrit -oire(s) ${ }^{34}$.

Comme on le voit, il s'agit ici de ce que traditionnellement l'on considère comme une "variante graphique". Mais lorsque certaines graphies sont récurrentes, elles peuvent nous donner des indications intéressantes, surtout si elles contribuent à compléter un faisceau d'éléments d'autre nature. Le suffixe -ore pour -oire est un trait que l'on considère généralement comme typique du Nord de la France (Zink, 1986 : 197), voire plus spécifiquement comme picard (Gossen, 1970 : 82-83). S’il est vrai que cette graphie est particulièrement représentée dans le domaine picard, on en trouve des attestations aussi ailleurs dans le domaine d'oïl.

Dans le DMF 2015, la graphie victore est attestée uniquement chez Froissart, quatre fois. La graphie glore (s) y est attestée onze fois, toujours dans des textes d'origine picarde : en plus de Froissart, l'Internele consolacion ( $c$ 1447) et Le Somme abregiet de theologie (ca 1477-1481). Nous avons étendu notre recherche aux mots histoire et

\footnotetext{
${ }^{31}$ Ainsi dans l'autre MO P1 (les chapitres en question aux f. 38vb-39va, 90va-91vb, 156vb-158ra, mais le chap. 25, qui correspond au chap. 21 de la $V 1$ ', manque dans ce manuscrit) et dans les MT Lo (f. 39vb40vb, 43ra-45ra, 96vb-98ra, 171rb-172vb) et Pa4 (f. 19r-19v, 20v-21v, 54r-54v, les derniers chapitres de la troisième partie, dont 3.19 donc, manquent dans ce manuscrit).

32 Comme ailleurs, les autres différences entre la $V 1$ et la $V 1$ ' ne sont pas commentées.

${ }^{33}$ Les chapitres concernés se trouvent aux f. 22r-23v, 56v-57r, 99v-100r.

${ }^{34}$ F. 34r-36v, 93v-95r, 171r-171v.
} 
mémoire. On trouve quatre attestations de la graphie (h)istore(s) dans le DMF 2015 : une attestation est dans Hugues Capet, une chanson de geste que le DMF date de 1358 et qui est conservée dans un seul manuscrit d'origine picarde. Mais les trois autres se trouvent respectivement dans Florence de Rome, un texte qui, d'après la notice du DMF, est édité d'après un manuscrit du XIV ${ }^{\mathrm{e}}$ siècle en scripta lorraine ${ }^{35}$; dans l'Inventaire $d u$ mobilier de Charles V, dont le DMF ne donne pas la date (Charles V est mort en 1380), mais qui aurait été écrit en "milieu curial " d'après la notice; et enfin dans La Sale d'Antoine de la Sale, d'après l'édition de Desonay (1941: 21). Cette édition se base sur le manuscrit conservé à la Bibliothèque royale de Belgique sous la cote 10959, daté de $1451^{36}$. Memore, quant à lui, est attesté deux fois : dans le même Somme abregiet de theologie dont il vient d'être question, mais aussi dans le Cartulaire de l'Hôtel-Dieu de Coutances pour l'année 1478. Coutances se trouve en Normandie (département de la Manche).

Une origine picarde, que ce soit pour l'archétype de la $V 1$ 'ou pour l'antigraphe de $\mathrm{Mu}$ et de $\mathrm{Pa} 1$, semble à exclure, car aucun des témoins de cette version ne présente de traces de la scripta picarde, encore assez caractérisée au $\mathrm{XV}^{\mathrm{e}}$ siècle. Une origine plus largement septentrionale, en revanche, ne serait pas en contradiction avec le lieu de production des manuscrits. Mais, en plus du fait qu'un seul trait linguistique ne peut pas être considéré comme un indice fiable pour la localisation d'un manuscrit, dans notre cas spécifique, étant donné la correspondance exacte entre $\mathrm{Mu}$ et $\mathrm{Pa} 1$ et leur désaccord avec Wi, la graphie pourrait trahir les habitudes du copiste du modèle commun aux deux premiers manuscrits, dont l'existence a déjà été évoquée ${ }^{37}$.

Si le suffixe -ore était le fruit d'innovations graphiques indépendantes, il serait étonnant de se trouver devant un accord si parfait entre $\mathrm{Mu}$ et Pa1. L'emploi

\footnotetext{
${ }^{35}$ Nous ferons remarquer en passant que la forme memore apparaît aussi dans un manuscrit beaucoup plus ancien copié en scripta lorraine, manuscrit qui contient la traduction française des sermons de Bernard de Clairvaux et qui daterait de la fin du XII ${ }^{\mathrm{e}}$ ou du début du XIII ${ }^{\mathrm{e}}$ siècle (Foerster 1885: 121 . 1 et 4, 26 1. 35, et passim ; sur le manuscrit, voir Careri, Ruby et Short, $2011: 168-169)$ ). Ce même manuscrit contient aussi de nombreuses attestations de la forme glore (Foerster 1885: $51.23,111.29$ et 35, et passim).

${ }^{36}$ Nous n'avons pas procédé à un examen attentif de la scripta de ce copiste : d'après un rapide survol, elle ne semble pas spécialement picardisante, mais il est vrai que le manuscrit fut copié pour Louis de Luxembourg, dont les terres étaient situées principalement dans le domaine linguistique picard (sur ce manuscrit, on verra Lefevre, 2006 : 415-416). À titre de curiosité, nous faisons remarquer que dans le texte on trouve aussi les graphies « régulières" memoire et victoires (Desonay, 1941:104).

${ }^{37}$ Les témoins de la $V x$, dont l'archétype, nous le savons, a été en partie copié sur $\mathrm{Mu}$, ne peuvent pas vraiment nous aider sur ce point, car des quatre chapitres du corpus dans lesquels on trouve des attestations du suffixe -ore, seul un, le chap. 2.29, a été copié d'après la VI' (les autres ayant pour modèle la $V 2$ ), et dans ce chapitre il y a une seule occurrence de ce suffixe : les trois manuscrits consultés écrivent victoires ( $\mathrm{Pr}$ f. 121v, Pa2 f. 78va et Pa8 f. 62r). Mais cela n'est pas étonnant, si l'on pense qu'entre $\mathrm{Mu}$ et les témoins de la $V x$ il y a eu au moins leur archétype, dont le copiste avait une tendance manifeste à innover.
} 
systématique de la graphie -ore semble bien être imputable à leur antigraphe commun. L'on serait donc en présence d'un cas où une habitude linguistique confirme les observations philologiques. Cette habitude a par ailleurs pu être adoptée par les copistes de $\mathrm{Mu}$ et de Pal parce qu'elle n'allait pas à l'encontre de leurs propres habitudes. Ainsi, même en admettant que la graphie -ore puisse nous orienter vers la Normandie, elle ne peut pas nous aider à situer géographiquement l'auteur du remaniement, car vraisemblablement celui-ci ne l'utilisait pas, ou pas systématiquement : s'il l'avait utilisée systématiquement, elle aurait probablement laissé des traces dans Wi, comme le suggère aussi le comportement de son copiste quand on regarde à d'autres traits graphiques, tel celui qui sera analysé dans le prochain paragraphe. Mais bien entendu, le fait que le remanieur à l'origine de la $V 1$ ' n'ait pas utilisé la graphie -ore ne signifie pas que celuici n'ait pas pu écrire en Normandie, car ce trait graphique d'une part était loin d'être circonscrit à cette région, et d'autre part ne semble pas y avoir été aussi répandu qu'en picard $^{38}$, sans parler du fait qu'aucun des traits caractéristiques des différentes scriptas n'est utilisé systématiquement par les copistes.

\subsection{La P3 du passé simple du verbe avoir : un ténu indice diatopique (et diachro- nique ?)}

Notre dernier point montrera, tout comme le précédent, comment les traits linguistiques peuvent appuyer les observations philologiques; il confirmera également que les copistes avaient tendance, au moins dans une certaine mesure, à adopter les choix graphiques de leurs modèles. Nous nous intéresserons à la P3 du passé simple du verbe avoir.

Dans les MO de Christine de Pizan, cette forme se présente essentiellement sous la forme $o t$ : on en trouve de nombreux exemples dans tous les manuscrits, aussi bien de la $V 1$ que de la $V 2$. Si l'on regarde à l'édition de la $V 1$ 'd'après $\mathrm{Mu}$, on remarque que l'on n'a jamais la forme $o t$. La forme utilisée est toujours olt dans la première partie et, dans notre corpus, deux fois dans la deuxième partie ; mais dans la deuxième partie, la forme de la $\mathrm{P} 3$ du passé simple est de préférence eust.

Tout ceci peut sembler anecdotique, mais il l'est peut-être moins quand on compare les formes de $\mathrm{Mu}$ avec celles que l'on trouve dans les autres témoins de la $V 1$ ',

\footnotetext{
${ }^{38}$ Sauf erreur, le suffixe -o(i)re n'est mentionné ni dans l'ouvrage de Goebl (1970) sur la scripta des chartes normandes, ni dans les études de Pope (1952) et de Short (2013) sur l'anglo-normand, qui partage des traits avec sa variante continentale, ni dans le volume de Chaurand (1972) sur la dialectologie française ; des mots susceptibles de nous intéresser ne se trouvent pas non plus dans les cartes de l'Atlas linguistique de la France.
} 
$\mathrm{Pa} 1$ et $\mathrm{Wi}^{39}$, ainsi qu'avec l'un des témoins de la $V x, \mathrm{~Pa} 2^{40}$, la rédaction " contaminée " dont l'archétype a probablement été en partie copié, comme on le sait, sur Mu luimême ou sur un manuscrit très proche de celui-ci. La régularité de la distribution des formes dans les manuscrits est frappante :

\begin{tabular}{|l|c|c|c|c|}
\cline { 2 - 5 } \multicolumn{1}{c|}{} & Mu & Pa1 & Wi & Pa2 \\
\hline 1.1 .9 & olt & olt & oult & ot \\
\hline 1.1 .27 & olt & olt & oult & ot \\
\hline $1.21 .5^{\text {I }}$ & olt & olt & olt & ot \\
\hline $1.21 .5^{\text {II }}$ & olt & olt & olt & manque \\
\hline 1.21 .9 & olt & olt & olt & avoit \\
\hline 1.21 .12 & olt & olt & olt & manque \\
\hline 1.21 .17 & olt & olt & olt & manque \\
\hline 1.21 .20 & olt & olt & ot & manque \\
\hline 1.21 .26 & olt & olt & olt & ot \\
\hline 1.22 .8 & olt & olt & olt & ot \\
\hline 2.29 .11 & eust & eust & eust & eust \\
\hline 2.29 .23 & eust & eust & eust & eust \\
\hline 2.29 .24 & eust & eust & eust & eut \\
\hline 2.29 .25 & olt & eust & eust & ot \\
\hline 2.53 .2 & olt & eust & eust & ot \\
\hline 2.53 .16 & eust & eust & eust & eut \\
\hline
\end{tabular}

Il faut d'abord préciser que, dans la $V x$ (représentée ici par Pa2), les chap. 1.211.22 ont été copiés d'après la $V 2$ et non pas d'après la $V 1$ '. Par conséquent, dans un cas, $\mathrm{Pa} 2$, comme la $V 2$, a l'imparfait et non pas le passé simple (1.21.9); dans quatre occurrences, les versions ne sont pas comparables, car la $V x$ reformule $\left(1.21 .5^{\mathrm{II}}\right.$, $1.21 .12,1.21 .17$ et 1.21 .20 , mais sur ce dernier passage on verra la note correspondante) ; dans les autres occurrences $\left(1.21 .5^{\mathrm{I}}, 1.21 .26\right.$ et 1.22 .8$)$, la forme du passé simple est $o t$. Certes, on trouve cette forme aussi ailleurs dans $\mathrm{Pa} 2$, mais toujours quand il y a olt dans $\mathrm{Mu}$; en revanche, $e u(s) t$, pour le passé simple, ne semble pas être une forme propre au copiste de $\mathrm{Pa} 2$, qui manifestement l'utilise uniquement quand il la trouve dans son modèle.

\footnotetext{
${ }^{39}$ Les chapitres où l'on trouve les paragraphes répertoriés dans le tableau ci-dessous se trouvent respectivement aux f. 4ra-5va, 25rb-27ra, 60vb-61va, 77rb-78va (Mu), 2r-3v, 22r-23v, 56v-57r, 72v-74r (Pa1) et $3 \mathrm{r}-5 \mathrm{r}, 34 \mathrm{r}-36 \mathrm{v}, 93 \mathrm{v}-95 \mathrm{r}, 123 \mathrm{v}-125 \mathrm{v}$ (Wi).

${ }^{40}$ Comme on le verra, les données fournies par ce témoin sont déjà suffisamment intéressantes pour qu'il n'ait pas été nécessaire d'étendre la vérification à d'autres témoins de cette version. Les chapitres vérifiés se trouvent aux f. 4rb-5vb, 29va-31va, 77va-78vb, 102ra-103va.

${ }^{41}$ Alors que dans les $₫ 1.21 .5$ (deuxième occurrence), 1.21.12 et 1.21.17 le texte est remanié aussi dans les autres témoins, dans ce paragraphe l'absence du passé simple est un trait particulier à Pa2 : Pr et Pa8, sur lesquels a été faite la vérification, ont ot (respectivement f. 63v et $28 \mathrm{v}$ ).
} 
Si l'on passe à la comparaison des manuscrits de la $V 1$ ', on remarque que presque toujours, quand $\mathrm{Mu}$ utilise la graphie olt pour le passé simple, cette graphie se retrouve telle quelle dans Pa1, et telle quelle ou sous les variantes oult (deux fois) ou ot (une fois) dans Wi (passages 1.1.9 à 1.22.8).

Mais dans la deuxième partie de la $C D, \mathrm{~Pa} 1$ et Wi notent toujours le passé simple par la graphie eust. Eust est aussi la graphie de Mu dans 4 des 6 occurrences de cette forme verbale dans les chapitres retenus de la deuxième partie. Les deux autres fois, $\mathrm{Mu}$ emploie la graphie olt (2.29.25 et 2.53.2). Pa2, quant à lui, utilise la graphie eust ou sa variante sans $s$ eut là où $\mathrm{Mu}$ a eust, et, comme dans la première partie, ot quand $\mathrm{Mu}$ écrit olt. Les occurrences ne sont pas très nombreuses, mais leur régularité est trop parfaite pour pouvoir être attribuée au hasard, sans compter que des vérifications faites sur d'autres échantillons dans le texte ont confirmé les résultats obtenus ici.

Ces vérifications ont été faites sur les trois témoins de la VI' dans des chapitres choisis au hasard dans les trois parties, chapitres dans lesquels la P3 du passé simple du verbe avoir revient dans les premières lignes. Dans la première partie, les trois manuscrits ont systématiquement la graphie olt (oult Wi dans le chap. 1.30) ; dans les deuxième et troisième parties, ils ont systématiquement eust. Voici les données :

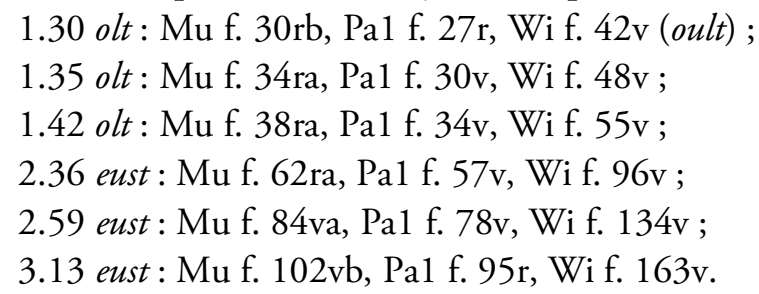

Puisque les deux graphies olt et eust se retrouvent de manière systématique dans les représentants de la $V 1$ ' avec une distribution régulière, il est fort probable qu'elles aient été présentes dans l'archétype de cette version. Pal et Wi s'accordent entre eux pour employer la graphie olt (avec les variantes oult et, une seule fois, ot pour le deuxième) dans la première partie, et eust dans la deuxième partie. Quant à $\mathrm{Mu}$, la graphie olt y réapparait, sporadiquement, dans la deuxième partie : peut-être cette graphie avait-elle la préférence de son copiste, qui a eu tendance à la substituer à la graphie qu'il trouvait dans son modèle ${ }^{42}$. La raison pour laquelle, avec toute vraisemblance, il y avait deux graphies pour le même morphème dans l'archétype du remaniement nous est inconnue, mais nous reviendrons sur ce point.

On remarquera que dans tous les témoins de la $V 1$ 'on trouve d'autres graphies de passés simples avec des -s- non étymologiques, et cela, dès le début du texte. Dans

\footnotetext{
${ }^{42}$ Nous ne saurions affirmer que cette modification supplémentaire dans Mu serait à mettre en relation avec le remplacement de mie par pas, toujours de manière non systématique, également dans la deuxième partie. Certes, ce n'est pas impossible d'y voir un rapport (un changement de copiste ?), mais sur la difficulté d'attribuer le remplacement de mie au copiste de Mu lui-même plutôt qu'à celui d'un antigraphe, on verra ce qui a été écrit dans la section 3.1.
} 
quelques cas, quand $\mathrm{Mu}$ a une graphie en -s-, il arrive que d'autres témoins, et surtout Wi, emploient des graphies asigmatiques mais, au total, les cas où tous les manuscrits s'accordent pour offrir des formes sigmatiques sont assez nombreux pour penser que ces formes peuvent remonter à l'archétype.

Dans notre corpus, on trouve les exemples suivants ${ }^{43}$ :

1.2.8 creust $\mathrm{Mu}$ Pa 1 crust $\mathrm{Wi}$

1.21.9 sceust $\mathrm{Mu} \mathrm{Pa} 1$ sceut $\mathrm{Wi}$

2.29.7 creust $\mathrm{Mu}$ Pa1 creut Wi

2.29.8 congnust $\mathrm{Mu} \mathrm{Pa} 1$ congneust $\mathrm{Wi}$

2.29 .23 creust $\mathrm{Mu}$ Wi crust $\mathrm{Pa} 1$

2.29.26 crust $\mathrm{Mu}$ Pa1 creust Wi

À ces passés simples on peut ajouter la $\mathrm{P} 3$ du verbe $v(e)$ oir $:$ au $\$ 1.22 .8$ les trois manuscrits ont vist, au $\$ 2.29 .10$ vist est dans $\mathrm{Mu}$ et Pa1, Wi a vit. Dans tous ces cas, le manuscrit de base de la VI, A, a les formes asigmatiques (cr(e)ut, sceut, congneut, vit $)^{44}$.

Des vérifications par échantillons en dehors de notre corpus ont donné les résultats suivants ${ }^{45}$ :

1.3 congnust $\mathrm{Mu}$ congnut $\mathrm{Pa} 1$ congneust $\mathrm{Wi}$

2.60 vist... sceust... vist $\mathrm{Mu} \mathrm{Pa} 1$ vit... sceut... manque Wi

2.62 deust $\mathrm{Mu} \mathrm{Pa} 1 \mathrm{Wi}$

3.15 peust $\mathrm{Mu}$ Pa1 polt $\mathrm{Wi}$

3.15 receust $\mathrm{Mu}$ Pal receut $\mathrm{Wi}$

Certes, les exemples ne sont pas très nombreux, et surtout Wi a tendance à utiliser des formes asigmatiques plus souvent que les autres témoins; mais d'une part nous avons déjà vu que ce manuscrit montre une certaine indépendance à tous les niveaux, d'autre part il utilise aussi des formes en -s- non étymologiques qui sont totalement absentes, pour les passés simples, des MO.

Les graphies sigmatiques, y compris pour le verbe avoir, auraient-elles été spécifiques à un premier manuscrit (ms. 1), à identifier peut-être avec la copie du remanieur ? Le copiste d'un deuxième manuscrit aurait-il remplacé la graphie eust du passé simple par la graphie olt, qui lui était peut-être plus familière (ms. 2) ? Cette situation serait reflétée par Pal et par Wi. Le copiste de $\mathrm{Mu}$ se serait alors borné à éliminer quelques autres graphies sigmatiques. Soit, schématiquement :

\footnotetext{
${ }^{43}$ Les feuillets dans lesquels on trouve ces passages sont les suivants : Mu : 1.2 f. 5va-6va, 1.21-22 f. 25rb27ra, 2.29 f. 60vb-61va ; Pa1 : 1.2 f. 3v-4r, 1.21-22 f. 22r-23v, 2.29 f. 56v-57r ; Wi : 1.2 f. 5r-6r, 1.2122 f. 34r-36v, 2.29 f. $93 \mathrm{v}-95 \mathrm{r}$.

${ }^{44} 1.2$ f. 2vb-4ra, 1.21 (=1.22 V1) f. 30ra-30vb, 1.25 (=1.21 V1) f. 32rb-33vb, 2.29 f. 77ra-78rb.

${ }^{45} \mathrm{Mu}: 1.3$ f. 6va-7vb, 2.60 f. 84va-85va, 2.62 f. 85vb-86rb, 3.15 f. $103 \mathrm{va}-104 \mathrm{rb}$; Pa1 : 1.3 f. 4v-5v, 2.60 f. 80 rv, 2.62 f. 81 r, 3.15 f. 97 rv ; Wi : 1.3 f. 6v-8r, 2.60 f. 135v-137r, 2.62 f. 138rv, 3.15 f. 166v$167 \mathrm{v}$.
} 
ms. 1 eust

ms. 2 olt/eust (= Pa1 / Wi)

$\mathrm{Mu}$ olt/eust/olt

Mais pourquoi le copiste du ms. 2 aurait-il remplacé cette graphie uniquement dans la première partie ? Peut-il avoir de l'importance le fait que la graphie eust apparaît une fois dans la première partie dans un titre, celui du chap. 3 ? On sait que les titres rubriqués étaient souvent ajoutés après la transcription du texte, par le même copiste qui avait copié celui-ci ou par un autre scribe.

Cy dit Cristine que la dame qui l'eust arraisonnee lui dist quelle estoit sa proprieté et de quoy elle servoit... (Mu f. 6va, Pa1 f. 4v, Pa2 f. 7ra ; dans Wi, les titres des chapitres n'ont pas été copiés.)

A-t-il existé un ms. 2, ou bien y a-t-il eu simplement un changement de copiste lors de la copie du ms. 1 ? Mais si tel est le cas, celui-ci coïnciderait difficilement avec la copie du remanieur, car les modalités du remaniement, bien qu'elles se réduisent le plus souvent à une banalisation, ne changent pas d'une partie à l'autre. Il est alors possible qu'il y ait eu un changement de scribe lors de la copie du ms. 2. Quoi qu'il en soit, derrière le sigle $V 1$ ', dans le stemma codicum proposé dans l'Annexe 2, il faut probablement voir plusieurs manuscrits.

Nous avons vu que les copistes pouvaient varier les graphies de certains mots dans un but rhétorique: mais le principe de la démonstration de Llamas-Pombo (2017 : 48) est que la recherche de la variatio était faite à proximité, ce qui n'est pas le cas ici. Quoi qu'il en soit, un copiste semble ici avoir changé de graphie ou, plus probablement (?), un manuscrit a changé de copiste après la première partie. Mais ce qu'il importe de faire remarquer, c'est que, comme nous l'avons déjà vu pour la graphie orel-oire, les scribes pouvaient avoir tendance à copier de leur modèle aussi le système graphique.

Ces graphies sigmatiques de passé simple, qui ont des chances de remonter au remanieur, peuvent-elles nous aider à dater et à localiser son travail ? Et qu'en est-il de la forme olt, qui, si elle ne remonte pas au remanieur, a sans doute été employée dans un manuscrit qui précède au moins aussi $\mathrm{Mu}$ ? Une recherche dans les textes du $\mathrm{XV}^{\mathrm{e}}$ siècle de Frantext a montré que le morphème olt y est enregistré pour la première fois dans les Mémoires d'Olivier de la Marche, datés de 1470. La forme co(n)gnust, elle, y est attestée pour la première fois dans un texte de Jean Juvenal des Ursins, Verba mea auribus percipe, Domine, daté de 1452. Mais un autre passé simple en -s- non étymologique, vist, serait attesté dans les Enfances Garin de Monglane, daté de 1400, alors qu'il n'y a pas d'attestation de crust.

Les attestations de eust, elles, se comptent par milliers au $\mathrm{XV}^{\mathrm{e}}$ siècle ${ }^{46}$, car eust était aussi, et surtout, la forme de la P3 du subjonctif imparfait. Nous n'avons pas poussé plus loin les recherches dans Frantext, car nous savons que des recherches sur les graphies sont très délicates à mener dans cette base de données (qui, dans d'autres

${ }^{46} 2834$ occurrences dans les 160 textes compris entre 1400 et 1499. 
domaines, est on ne peut plus précieuse). Les textes enregistrés sont parfois tirés d'éditions très anciennes, mais surtout, ce qui est renseigné c'est la date du texte, et non pas, ou pas nécessairement, la date du manuscrit de base ${ }^{47}$.

Les graphies en -s- non étymologiques pour les passés simples semblent avoir existé dès au moins le début du $\mathrm{XV}^{\mathrm{e}}$ siècle. Christine de Pizan a pu les utiliser ellemême, mais, semble-t-il, essentiellement en rime, comme dans la Complainte qui termine le Livre du Duc des vrais amants :

Car qu'il morust

Qui tost ne le secourust

Sembloit, plus roide que un fust,

Et que l'eaue li courust

En redoublant

Sus son vis, et qu'il ne peust

Parler. Si grant douleur eust

Si qu'au lit de tous poins geust,

Mort ressemblant

(Demartini et Lechat, 2013 : 414-416, v. 56-64)

Ces graphies semblent avoir été peu usitées auprès des copistes parisiens, ou en tout cas auprès des copistes auxquels Christine de Pizan avait l'habitude de s'adresser. L'auteure n'avait vraisemblablement pas l'habitude de les utiliser elle-même, car autrement il est probable qu'elles apparaîtraient quelque part dans les MO, étant donné que, comme nous venons de le voir, les copistes avaient tendance à reproduire, au moins en partie, les graphies de leurs antigraphes.

Au demeurant, la graphie olt pour la P3 du passé simple est attestée en Normandie dès la fin du XIV ${ }^{\mathrm{e}}$ siècle (Goebl, $1970: 313^{48}$ ). Si cette observation ne nous aide pas à dater le remaniement, elle pourrait nous aider à le localiser. Mais si nous avons vérifié ces graphies dans la scripta usitée en Normandie, c'est d'une part parce qu'il existe une étude sur celle-ci, et d'autre part parce que nous les y avons cherchées, étant donné que la $V 1$ ' semble avoir joui d'un certain succès dans cette région de France ; les mêmes graphies, toutefois, peuvent se rencontrer ailleurs.

Sauf erreur de notre part, la forme olt et la forme eust ne sont décrites ni dans Pope (1952), ni dans Gossen (1970), ni dans Remacle (1948), pour ne citer que trois études approfondies de scriptas régionales (anglo-normand, picard, wallon). Mais une vérification dans le Nouveau corpus d'Amsterdam (Stein et al., 2006) a montré que la

\footnotetext{
${ }^{47}$ Dans la BFM, les occurrences sont 925, et donc plus maîtrisables ; mais, dans cette base de données aussi, les manuscrits ne sont pas toujours datés ni localisés, surtout pour les textes empruntés à des éditions anciennes, ce qui rend peu utile un travail mené sur les graphies. Nous en profitons pour faire remarquer que, sauf erreur de notre part, il n'y a pas d'attestations de la graphie olt dans les textes du $\mathrm{XV}^{\mathrm{e}}$ de la BFM.

${ }^{48}$ À Longueville (Calvados) en 1388. Dans la même charte, Goebl signale aussi l'emploi de oult.
} 
forme olt serait attestée dès la première moitié du XIII ${ }^{e}$ siècle à Liège : " ...com il olt grazes rendues a Deu del chiel " (d'après Pasquet, 1888: $38^{49}$ ). Par ailleurs, Short $(2013$ : 138, \$ 34.9) signale des formes de passés simples en $-i$ (mais non pas en $-u$ ) avec un $s$ non étymologique pour assurer la rime dès... le XII ${ }^{\mathrm{e}}$ siècle ${ }^{50}$ !

Une étude approfondie des graphies usitées pour les passés simples d'une part à Paris et d'autre part dans des régions autres que la Normandie n'a pas été tentée pour la présente étude. Une telle étude devrait se fonder sur un corpus de manuscrits préalablement datés et localisés de manière sûre, mais elle allait au-delà des limites fixées pour ce travail.

\section{Conclusions}

Que faut-il conclure de cette étude sur certains morphèmes et certaines graphies de l'une des versions de la $C D$ qui ne remonte assurément pas à l'auteure et qui n'est conservée que dans des MT ?

D’une part, on a eu confirmation, si besoin était, que les copistes pouvaient reproduire, dans une certaine mesure, les graphies des manuscrits qu'ils copiaient. Cela devait être d'autant plus vrai quand le système graphique du modèle n'était pas en

\footnotetext{
${ }^{49}$ Nous remercions Frédéric Duval de nous avoir signalé cette occurrence. La section du manuscrit dans laquelle sont contenus les Sermons de Carême, d'où est tirée la citation (Bibliothèque de l'Université de Gand, HS.2178), est datée de la première moitié du XIII ${ }^{e}$ siècle dans Pasquet (1888: 4-5). Derolez (2017 : 257) date cette section du XIII siècle, en ajoutant toutefois que ce manuscrit composite mériterait une étude plus approfondie. Nous avons pu vérifier une seule page de ce codex, qui n'est pas numérisé, grâce à l'obligeance de Madame Femke Van der Fraenen, conservatrice à la Bibliothèque de l'Université de Gand, qui a bien voulu nous en fournir gracieusement une photographie : s'il n'est pas certain que le manuscrit soit aussi ancien, il semble sûr qu'il est bien antérieur à la fin du XIV ${ }^{e}$ siècle. La scripta du texte semble en effet être wallonne, et le manuscrit a d'ailleurs appartenu à l'abbaye de SaintJacques à Liège.

${ }^{50}$ L'impression est néanmoins que la graphie eust pour la P3 du passé simple du verbe avoir, comme d'autres graphies sigmatiques non étymologiques, se répandent plus nettement vers le milieu du $\mathrm{XV}^{\mathrm{e}}$ siècle. Ainsi, par exemple, sont-elles nombreuses dans le Jehan de Saintré d'Antoine de la Sale tel qu'on le lit dans le manuscrit BnF, n.a.f. 10057, copié en mars 1456 (Lefevre, 2006 : 421) : fust, sceust, eust, oÿst, etc. (Blanchard et Quereuil, 1995 : 36-54 et passim). Dans le Testament de Villon du manuscrit BnF, fr. 20041, datable vers 1467-1471, eust est employé cinq fois, bien que ot y apparaisse encore huit fois : eust v. 275, 285, 340, 1888 et 1895 ; ot 153, 335, 366, 426, 662, 867, 1024 et 1211 (à lire aujourd'hui de préférence dans Cerquiglini-Toulet, 2020 ; le point sur le manuscrit, très étudié, aux p. 4648). Mais, bien entendu, de telles affirmations devraient être vérifiées sur des comptages systématiques menés sur des corpus bien plus vastes. Nous rappellerons, pour terminer, que Marchello-Nizia (1997: 282) ne parle pas des formes sigmatiques, mais s'intéresse au remplacement du radical $o$ par $e u$ aux P1, $\mathrm{P} 3$ et $\mathrm{P} 6$ du verbe avoir et assimilés (comme $o t>e u t$ ) : d'après elle, les formes en $e u$ sont partout majoritaires au $\mathrm{XV}^{\mathrm{e}}$ siècle, mais, si elles alternent avec les forme en $o$ dans la première moitié du siècle, on n'aurait que des formes en $e u$ chez Charles d'Orléans (mort en 1465 et dont on possède un manuscrit partiellement autographe : voir Arn, 2008), Saintré et les Quinze joies de mariage (éditées d'après un manuscrit daté de 1464, d'ailleurs copié à Rouen : voir Rychner, 1963 : LIX).
} 
contradiction avec celui de la copie, comme c'est probablement le cas pour-orel-oire; ou bien, à l'inverse, les formes graphiques pouvaient être d'autant plus conservés qu'elles étaient plus rares, ce qui se produit peut-être pour les graphies sigmatiques des passés simples. L'étude des graphies, en plus d'être utile aux linguistes, ne doit donc pas être négligée par les philologues, car elle peut apporter au moins des confirmations à des résultats atteints via l'analyse des leçons.

Certes, les copistes ne reproduisaient pas toujours les graphies qu'ils trouvaient dans leur modèle, car autrement les graphies de la $V 1$ ' seraient encore celles des $\mathrm{MO}$ de Christine de Pizan. On sait que certains copistes étaient plus entreprenants que d'autres ; cet "esprit d'initiative " pouvait s'exercer aussi dans des faits graphiques. Il n'est ainsi pas impossible que la même personne qui a remanié la $V 1$ pour aboutir à la $V 1$ ' en ait aussi considérablement revu l'aspect linguistique, ce qui au demeurant semble être certain pour ce qui concerne le remplacement de certains morphèmes par d'autres (comment > comme, tout soit $>$ tant soit). De la même manière, il n'est pas surprenant que les différentes copies de la $V x$, tout en gardant des traces des graphies de $\mathrm{Mu}$, ne les reproduisent pas fidèlement, étant donné que, en plus des innovations singulières des copistes des différents manuscrits, il faut tenir compte des innovations sans doute introduites par le copiste à l'origine de l'archétype : celui-ci devait être un manuscrit très remanié, ne serait-ce que parce que la $V 1$ 'y a été croisée avec la $V 2$.

D'autre part, nous pouvons dresser des conclusions, hélas minces et peu concluantes, sur les tentatives de datation et de localisation de l'archétype du remaniement $V 1$ '. Nous avons vu que le remplacement, non systématique mais assez constant, de mie par pas est typique uniquement de $\mathrm{Mu}$; l'emploi systématique du suffixe -ore pour -oire, lui, remonte probablement à l'antigraphe de $\mathrm{Mu}$ et de Pa1. Seuls les remplacements de tout concessif par tant et de comment par comme semblent remonter au remanieur. L'emploi des graphies sigmatiques pour les passés simples pourrait également lui être attribué. Un doute persiste sur la graphie olt, qui pourrait remonter au remanieur ou avoir été introduite dans une copie postérieure, mais antérieure à tous les témoins conservés.

L'emploi de comme ne semble pas pouvoir fournir des indications chronologiques. Tout semble être plus archaïque que tant, mais il pourrait avoir été déjà vieilli à l'époque de Christine de Pizan, ce qui fait que même un remanieur contemporain pourrait lui avoir préféré la forme tant. Les formes eust et olt pour le passé simple deviennent peut-être plus courantes après les premières décennies $\mathrm{du} \mathrm{XV} \mathrm{X}^{\mathrm{e}}$ siècle, mais elles ne sont pas inconnues au début du siècle, bien qu'elles ne semblent pas être très usitées à Paris à cette époque.

Le fait que Christine de Pizan semble avoir exercé un contrôle assez attentif sur les manuscrits de ses œuvres plaide toutefois en faveur du fait que le remaniement ait été composé loin de Paris, ou bien après la mort de l'auteure vers 1430, ou du moins 
après qu'elle s'est retirée dans une abbaye en $1418^{51}$. Il n'est pas impossible que son origine soit à situer en Normandie, plutôt vers la fin de la période considérée : les manuscrits qui conservent la $V 1$ 'ont été produits, certainement ou possiblement, en Normandie ; le témoin le plus ancien pourrait être postérieur à 1440 ; les graphies utilisées pour les passés simples dont témoignent tous les témoins sont attestées au moins aussi en Normandie et semblent devenir plus courantes à partir du milieu du siècle; tant concessif est une forme plus moderne que tout. Si la linguistique diachronique et la linguistique variationnelle ne peuvent pas à elles seules répondre à nos questions, croisées avec les données codicologiques et philologiques elles peuvent nous fournir des indications intéressantes.

Les dates post et ad quem de la VI' resteront donc ca 1406 (production d'un manuscrit issu de la $V 1$ utilisé par le remanieur) - années 1440 (production de Mu); sa localisation est incertaine. Mais, bien qu'elles soient très conjecturales, la localisation (Normandie) et la datation (années 1430-1440) suggérées dans la présente contribution, ne semblent pas être impossibles.

\section{RÉFÉRENCES BIBLIOGRAPHIQUES}

ARN, Mary-Jo (2008) : The Poet's Notebook: The Personal Manuscript of Charles d'Orléans (Paris BnF MS fr. 25458). Turnhout, Brepols (Texts \& Transitions, 3).

AUTRAND, Françoise (2009) : Christine de Pizan : Une femme en politique. Paris, Fayard.

$\mathrm{BFM}=$ Base de français médiéval, ENS de Lyon, URL : http://txm.bfm-corpus.org

BlanChARD, Joël [éd.] \& Michel Quereuil [trad.] (1995) : Antoine de La Sale, Jehan de Saintré. Paris, Librairie générale française (Lettres gothiques).

BROWN-GRANT, Rosalind (2000) : "Christine de Pizan : Feminist Linguist avant la lettre?", in John Campbell \& Nadia Margolis (éds), Christine de Pizan 2000 : Studies on Christine de Pizan in Honour of Angus J. Kennedy. Amsterdam, Rodopi (Faux titre, 196), 65-76.

BROWN-GRANT, Rosalind (2002) : "Writing Beyond Gender : Christine de Pizan’s Linguistic Strategies in the Defence of Women ", in Angus J. Kennedy (éd.), Contexts and Continuities. 3 vol., Glasgow, University of Glasgow Press, 1, 155-169.

CARAFFI, Patrizia [trad. italienne] \& Earl Jeffrey RICHARDS [éd.] (2003) : Christine de Pizan, La città delle dame. Milano, Luni, 1997 ; nouv. éd. Roma, Carocci (Biblioteca medievale, 2).

CARERI, Maria, Christine RUBY \& Ian SHORT (2011) : Livres et écritures en français et en occitan au XII siècle. Roma, Viella (Scritture e libri del medioevo).

${ }^{51}$ La biographie la plus récente de Christine de Pizan est due à Autrand (2009), mais elle n'ajoute rien de nouveau, surtout pour les données biographiques, à la brève mais précise étude de Solente (1969). 
Cazal, Yvonne, Gabriella Parussa, Cinzia Pignatelli \& Richard Trachsler (2003) : "L'orthographe : du manuscrit médiéval à la linguistique moderne ». Médiévales, 45, 99-118.

Cerquiglini-ToUlet, Jacqueline [éd. et trad.] (2020) : François Villon, Euvres complètes. Paris, Gallimard (Folio classique).

CHAURAND, Jacques (1972) : Introduction à la dialectologie française. Paris / Bruxelles / Montréal, Bordas.

CuRnow, Maureen Cheney (1975) : The Livre de la cité des dames of Christine de Pisan : A Critical Edition. Vanderbilt University, Nashville, Tennessee [thèse inédite].

Demartini, Dominique \& Didier LeChat [éds et trad.] (2013) : Christine de Pizan, Le Livre $d u$ duc des vrais amants. Paris, Champion (Champion Classiques. Moyen Âge, 37).

DeroleZ, Albert (2017) : Medieval Manuscripts : Ghent University Library. Ghent, Snoeck.

DesOnay, Fernand (1941) : Antoine de la Sale, CEuvres complètes, Tome II : La Sale. Liège I Paris, Faculté de Philosophie et Lettres / Droz.

DFM = Dictionnaire du Moyen Français (1330-1500). URL $:$ http://zeus.atilf.fr/dmf/

DugAZ, Lucien (2019) : Le Livre des fais d'armes et de chevalerie de Christine de Pizan. Édition critique. Thèse de doctorat sutenue à l'Université Sorbonne nouvelle Paris 3 sous la direction de Gabriella Parussa [à paraitre chez Classiques Garnier].

FOERSTER, Wendelin [éd.] (1885) : Li Sermon saint Bernart. Älteste französische Uebersetzung der lateinischen Predigten Bernhards von Clairvaux nach der Feuillantiner Handschrift in Paris. Erlangen, Deichert.

FOURNIER, Nathalie (1998) : Grammaire du français classique. Paris, Belin.

FRANTEXT = ATILF (1998-2021). Base textuelle Frantext (en ligne). ATILF-CNRS \& Université de Lorraine. URL : https://www.frantext.fr/

GoebL, Hans (1970): Die normandische Urkundensprache. Ein Beitrag zur Kenntnis der nordfranzösischen Urkundensprachen des Mittelalters. Vienne, Hermann Böhlaus Nachf.

GOSSEN, Carl Theodore (1967) : Französische Skriptastudien. Vienne, Hermann Böhlaus Nachf.

Gossen, Charles Théodore (1970) : Grammaire de l'ancien picard. Paris, Klincksieck.

GREUB, Yan (2018) : «La stratigraphie linguistique des manuscrits médiévaux et la variation linguistique ». Medioevo Romanzo, 42: 1, 6-30.

IBEAS VUELTA, Nieves (2020) : "Conciencia feminista, discurso literario y legitimación auctorial: Le Livre de la Cité des Dames de Christine de Pizan ". Çédille, revista de estudios franceses, 17, 243-265. DOI : https://doi.org/10.25145/j.cedille.2020.17.13.

JOHnston, Hope [éd.] (2014) : Christine de Pizan, The Boke of the Cyte of Ladyes, Translated by Brian Anslay. Tempe, Arizona Center for Medieval and Renaissance Studies (Medieval and Renaissance Texts and Studies, 457).

LAIGLE, Mathilde (1912) : Le Livre des trois vertus de Christine de Pisan et son milieu historique et littéraire. Paris, Champion (Bibliothèque du $\mathrm{XV}^{\mathrm{e}}$ siècle, 16). 
LANGE, Monika (1974) : Christine de Pisan: Livre de la cité des dames. Kritische Textedition auf Grund der sieben überlieferten "manuscrits originaux » des Textes. Universität Hamburg [thèse inédite].

LEFÈVRE, Sylvie (2006) : Antoine de la Sale : La fabrique de l'œuvre et de l'écrivain. Genève, Droz (Publications romanes et françaises, 238).

LLAMAS-POMBO, Elena (2017) : «Graphie et ponctuation du français médiéval. Système et variation », in Gabriella Parussa, Maria Colombo Timelli \& Elena Llamas-Pombo (éds), Enregistrer la parole et écrire la langue dans la diachronie du français. Tübingen, Narr Francke Attempto, 41-89.

LUSIGNAN, Serge (1999) : "Langue française et société du XIII ${ }^{e}$ au XV siècle ", in Jacques Chaurand (éd.), Nouvelle histoire de la langue française. Paris, Seuil, 91-143.

MarChello-Nizia, Christiane (1997 [1979]) : La langue française aux XIVe et XVe siècles. Paris, Nathan.

OuY, Gilbert, Christine Reno \& Inès Villela-PetiT (2012) : Album Christine de Pizan. Turnhout, Brepols (Texte, codex et contexte, 14).

PÄCHT, Otto \& Dagmar THOSS (1974) : Die illuminierten Handschriften und Inkunabeln der Österreichischen Nationalbibliothek. Französische Schule I. 2 vol., Vienne, Verlag der österreichischen Akademie der Wissenschaften.

PANTCHEVA CAPIN, Daniéla (2004) : « Le conservatisme de la langue, gage du caractère littéraire du texte et témoin d'une nouvelle conception de l'acte d'écriture : le cas d'Ysäje le Triste ». Medieum Avvum, 73 : 1, 66-92.

PARUSSA, Gabriella (2019) : «Imprimeurs et frontières linguistiques. Quel apport pour la périodisation linguistique ? ". Le français préclassique, 21, 75-96.

PASQUET, Emmanuel [éd.] (1888) : Sermons de carême en dialecte wallon : Texte inédit du XIII siècle. Bruxelles, F. Hayez.

POPE, Mildred K. (1952 [1934]) : From Latin to Modern French, with Especial Consideration of Anglo-Norman : Phonology and morphology. Manchester, Manchester University Press.

REMACLE, Louis (1948) : Le problème de l'ancien wallon. Liège, Faculté de Philosophie et Lettres.

RENO, Christine (2006) : "Les manuscrits originaux de la Cité des dames de Christine de Pizan ", in Tania Van Hemelryck \& Céline Van Hoorebeeck (éds), L'écrit et le manuscrit à la fin du Moyen Âge. Turnhout, Brepols (Texte, codex et contexte, 1), 267-276.

RICHARDS, Earl Jeffrey (2000) : "Editing the Livre de la cité des dames: New Insights, Problems and Challenges ", in Eric Hicks (éd.), "Au champ des escriptures": III colloque international sur Christine de Pizan. Paris, Champion (Études christiniennes, 6), 789816.

RYCHNER, Jean [éd.] (1963) : Les quinze joies de mariage. Genève, Droz (Textes littéraires français, 100).

SEGRE, Cesare (1979) : "Critica testuale, teoria degli insiemi e diasistema » [1976, 1978], in Semiotica e filologia. Testo e modelli culturali. Turin, Einaudi, 53-70. 
SHORT, Ian (2013 [2007]) : Manual of Anglo-Norman. Oxford, Anglo-Norman text society.

SOLENTE, Suzanne (1969) : Christine de Pisan. Extrait de l'Histoire littéraire de la France, 40, Paris, Imprimerie nationale / Klincksieck.

SOUTET, Olivier (1992) : La concession dans la phrase complexe en français des origines au XVI siècle. Genève, Droz (Publications romanes et françaises, 201).

STEIN, Achim et al. (2006) : Nouveau Corpus d'Amsterdam. Corpus informatique de textes littéraires d'ancien français (ca 1150-1350), établi par Anthonij Dees (Amsterdam 1987), remanié par Achim Stein, Pierre Kunstmann et Martin-D. Gleßgen. Stuttgart, Institut für Linguistik/Romanistik. URL: https://sites.google.com/site/achimstein/research/resources/nca.

VALENTINI, Andrea (2017) : "Encore. Quelques remarques sur les graphies des manuscrits de présentation : le cas des Epistres du debat sus le Rommant de la Rose », in Anna Loba (éd.), "Ton nom sera reluisant aprés toy par longue memoire" : Études sur Christine de Pizan. Posnan, Wydawnictwo Naukowe UAM, 71-81.

VALENTINI, Andrea (2019) : «La tradition manuscrite du Livre de la cité des dames de Christine de Pizan : sur la genèse et l'évolution d'un texte majeur du XV $\mathrm{XV}^{\mathrm{e}}$ siècle ». Romania, $137: 3-4,394-445$.

WeInREICH, Uriel (1954) : «Is A Structural Dialectology Possible ?». Word, 10, 388-400.

WILHELM, Raymund (2013) : «La copie d'un texte médiéval entre transcription et traduction : une nouvelle base de données pour la linguistique historique? ", in Raymund Wilhelm (éd.), Transcrire etlou traduire. Variation et changement linguistique dans la tradition manuscrite des textes médiévaux. Heidelberg, Universitätsverlag Winter (Studia romanica, 182), 1-13.

ZINK, Gaston (1986) : Phonétique historique du français. Paris, Presses universitaires de France.

\section{ANNEXE 1. LISTE DES TÉMOINS DU LIVRE DE LA CITÉ DES DAMES DE CHRISTINE DE PIZAN}

a) Manuscrits originaux

Première version $(V I)$

A : Paris, Bibliothèque de l'Arsenal, $2686\left(1405-1406^{52}\right)$.

P1 : Paris, BnF, fr. 24293 (1405-1406).

Deuxième version $(V 2)$

B : Bruxelles, KBR, 9393 (1407).

D : Paris, BnF, fr. 607 (1407-1408).

52 Certaines dates sont conjecturales; elles ont pour but surtout d'offrir une chronologie relative entre les manuscrits. Pour plus de précisions sur ces datations, voir Valentini (2019: 431-436). Certaines dates differrent légèrement de celles qui sont indiquées dans cette contribution, suite à des suppléments d'analyse dont on trouvera le détail dans l'ouvrage signalé dans la note 2 ci-dessus. 
L : Leiden, Universiteitsbibliotheek, Ltk 1819, fragment d'un feuillet (1408-1410 ?).

P2 : Paris, BnF, fr. 1179 (1406-1407).

P3 : Paris, BnF, fr. 1178 (1413-1415 ?).

R: London, BL, Harley 4431, deux volumes, vol. 2, f. 376r-398r (1410-1415).

\section{b) Manuscrits tardifs et volume imprimé}

V1

Engl : Bryan Anslay, Boke of the Cyte of Ladyes, London, Henry Pepwell, 1521 (traduction anglaise).

Cambridge, King's College Library, M.29.9.

London, BL, C.13.a.18.

London, BL, C.40.m.9.(12.) (fragment de 2 feuillets).

Longleat House Library, Wiltshire, STC 7271.

Oxford, Corpus Christi Library, $\triangle$.3.7.

Washington, D.C., Folger Shakespeare Library, STC 7271.

Lo: London, BL, Royal 19 A XIX (1425-1445).

Pa4 : Paris, BnF, fr. 826, f. 1r-84r, incomplet (milieu du XV siècle).

$V 2$

Br : Bruxelles, KBR, 9235-9237, f. 1r-132v (vers 1460-1470).

CV1 : Città del Vaticano, Biblioteca apostolica vaticana, Pal. Lat. 1966 (vers 14301445).

NH : New Haven, Yale University, Beinecke Library, 318 (milieu du XV ${ }^{e}$ siècle).

Pa5 : Paris, BnF, fr. 1177, f. 2r-112v (vers 1470-1480).

Pa6 : Paris, BnF, fr. 1182, f. 173r-254r (vers 1450-1460).

Pa7 : Paris, BnF, fr. 24292 (troisième quart du XV siècle).

Version remaniée anonyme (VI)

Mu : München, Bayerische Staatsbibliothek, Cod. gall. 8 (vers 1430-1450).

Pa1 : Paris, Bibliothèque de l'Arsenal, 3182 (vers 1460-1480).

Ph ?: ex-Phillipps 3648, collection particulière, f. 83r-150r, non consultable (troisième quart du XV siècle).

Wi : Wien, Österreichische Nationalbibliothek, 2605 (vers 1460-1480).

Version qui croise la $V 1$ ' et la $V 2(V x)$

Ch: Chantilly, Bibliothèque et archives du Musée Condé, 856 (562), texte très écourté, f. 1v-30r (vers 1450-1460).

CV2 : Città del Vaticano, Biblioteca apostolica vaticana, Reg. Lat. 918, f. 71r-132r (vers 1470-1480).

Ge : Genève, Bibliothèque de Genève, fr. 180 (troisième quart du XV siècle).

Li : Lille, Bibliothèque municipale, 390 (vers 1475-1485).

Pa2 : Paris, BnF, fr. 608 (troisième quart du XVe siècle).

Pa3 : Paris, BnF, fr. 609 (troisième quart du XV siècle).

Pa8 : Paris, BnF, fr. 24294, f. 1r-110r (troisième part du XV siècle).

Pr : Privas, Archives départementales de l'Ardèche, 7 (I 6), f. 25r-189v, incomplet (milieu du XV $\mathrm{XV}^{\mathrm{e}}$ siècle). 
Vlam : anonyme, Bouc van de Stede der Vrauwen, London, BL, Add. 20698 (vers 1475, traduction flamande).

ANNEXE 2. STEMMA CODICUM SIMPLIFIÉ DE LA VERSION REMANIÉE ANONYME (V1) DU LIVRE DE LA CITÉ DES DAMES

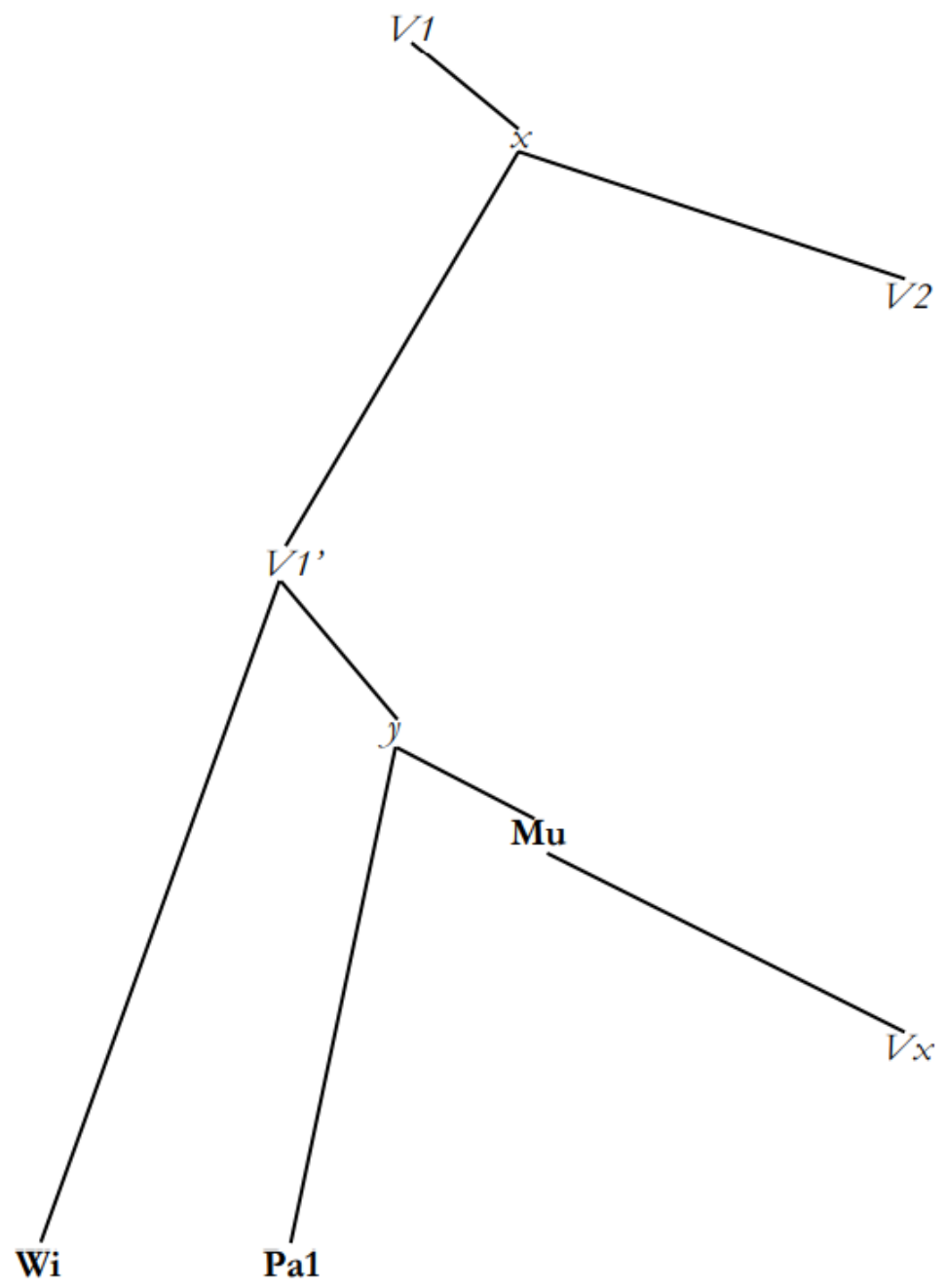

